\title{
Optimized Flat-Detector CT in Stroke Imaging: Ready for First-Line Use?
}

\author{
Matthias Eckert ${ }^{a}$ Philipp Gölitz ${ }^{a, b}$ Hannes Lücking ${ }^{a, b}$ Tobias Struffert ${ }^{a, b}$ \\ Frauke Knossalla ${ }^{a, c}$ Arnd Doerfler ${ }^{a, b}$ \\ ${ }^{a}$ Friedrich-Alexander-University-Erlangen-Nuremberg (FAU), ${ }^{b}$ Department of Neuroradiology, and ${ }^{\mathrm{c}}$ Department of \\ Neurology, University of Erlangen-Nuremberg, Erlangen, Germany
}

\section{Key Words}

Flat-detector CT · Stroke imaging · Image post-processing

\begin{abstract}
Background: Using flat-detector CT (FD-CT) for stroke imaging has the advantage that both diagnostic imaging and endovascular therapy can be performed directly within the Angio Suite without any patient transfer and time delay. Thus, stroke management could be speeded up significantly, and patient outcome might be improved. But as precondition for using FD-CT as primary imaging modality, a reliable exclusion of intracranial hemorrhage $(\mathrm{ICH})$ has to be possible. This study aimed to investigate whether optimized native FD-CT, using a newly implemented reconstruction algorithm, may reliably detect $\mathrm{ICH}$ in stroke patients. Additionally, the potential to identify ischemic changes was evaluated. Methods: Cranial FD-CT scans were obtained in 102 patients presenting with acute ischemic stroke $(n=32), \mathrm{ICH}(n=45)$ or transient ischemic attack $(n=25)$. All scans were reconstructed with a newly implemented half-scan cone-beam algorithm. Two experienced neuroradiologists, unaware of clinical findings, evaluated independently the FD-CTs screening for hemorrhage or ischemic signs. The findings were correlated to CT, and rater and inter-rater agreement was assessed. Results: FD-CT demonstrated high sensitivity (95$100 \%)$ and specificity (100\%) in detecting intracerebral and intraventricular hemorrhage (IVH). Overall, interobserver agreement $(\mathrm{K}=0.92)$ was almost perfect and rater agree-
\end{abstract}

\section{KARGER}

(c) 2016 S. Karger AG, Basel

E-Mail karger@karger.com

www.karger.com/ced ment to CT highly significant $(r=0.81)$. One infratentorial ICH and 10 or 11 of 22 subarachnoid hemorrhages (SAHs) were missed of whom 7 were perimesencephalic. The sensitivity for detecting acute ischemic signs was poor in blinded readings ( 0 or $25 \%$, respectively). Conclusions: Optimized FD-CT, using a newly implemented reconstruction algorithm, turned out as a reliable tool for detecting supratentorial ICH and IVH. However, detection of infratentorial $\mathrm{ICH}$ and perimesencephalic SAH is limited. The potential of FD-CT in detecting ischemic changes is poor in blinded readings. Thus, plain FD-CT seems insufficient as a standalone modality in acute stroke, but within a multimodal imaging approach primarily using the FD technology, native FD-CT seems capable to exclude reliably supratentorial hemorrhage. Currently, FD-CT imaging seems not yet ready for wide adoption, replacing regular $\mathrm{CT}$, and should be reserved for selected patients. Furthermore, prospective evaluations are necessary to validate this approach in the clinical setting.

(c) 2016 S. Karger AG, Basel

\section{Introduction}

Acute ischemic stroke management is developing rapidly. As gold standard intravenous (IV) rt-PA thrombolysis has been established, but there is now evidence that

M.E. and P.G. contributed equally to this paper. 
endovascular mechanical thrombectomy (EMT) improves outcome in comparison to sole IV thrombolytic therapy [1-3]. Besides careful neurological assessment, brain imaging is the major component within the diagnostic cascade in acute stroke. A native CT scan is necessary prior to IV rt-PA therapy to reliably exclude intracranial hemorrhage (ICH). Ideally, multimodal CT or MRI applying angiographic and perfusion imaging is performed to select patients who may take benefit from EMT $[4,5]$.

Any delay in stroke management should be avoided, since late recanalization does not improve outcome [6]. A delay of $30 \mathrm{~min}$ to revascularization decreases the probability of a good clinical outcome by more than 10\% [7]. Recent EMT studies report a median time up to $269 \mathrm{~min}$ from onset to start of EMT [8], which requires to be speeded up by workflow optimization.

Using flat-detector (FD) equipped angiographic systems, volumetric data can be acquired directly within the angiography suite allowing the reconstruction of crosssectional, CT-like images of FD-CT $[9,10]$. In combination with an IV contrast administration, FD-CT may provide high-resolution imaging of the cerebral vasculature and whole-brain perfusion in one acquisition as an 'onestop shop' stroke imaging [11]. Since 'time is brain', FD-CT may serve as an alternative imaging modality with the potential to significantly speed up stroke management, since diagnostic imaging as well as endovascular therapy can be performed in the identical setting avoiding patient transfer. However, a precondition to use FD-CT as primary imaging modality in acute stroke is the reliable hemorrhage exclusion [12]. So far, only a few studies regarding hemorrhage visualization by FD-CT are published, especially not in patients with acute ischemic stroke [13-15].

The aim of this study was to evaluate whether optimized FD-CT, using a newly implemented reconstruction algorithm, can reliably exclude hemorrhage in stroke patients. Additionally, the potential of FD-CT in identifying ischemic changes in patients with acute ischemic stroke was evaluated.

\section{Patients and Methods}

\section{Patients}

In a retrospective analysis, 102 patients - 54 men and 48 women - undergoing FD-CT of the brain were included, after informed consent was obtained. The average age of the patients was $63 \pm 12.5$ years (range $26-87$ years). Exclusion criteria were age $<18$ years, presence of an external ventricular drainage or any coil, clip or other metal implants (e.g., cochlear implants), which may cause significant beam hardening artifacts. All consecutive patients, examined by FD-CT in our department between December 2010 and January 2014, were included if no exclusion criteria were met. Before acquisition of the FD-CT data, an ethical study approval by the local institutional review board was obtained.

Clinically the patients presented with acute ischemic stroke $(\mathrm{n}=32)$, ICH $(\mathrm{n}=45)$ or transient ischemic attack (TIA; $\mathrm{n}=25)$.

ICH includes intracerebral, subarachnoid hemorrhage (SAH) and intraventricular hemorrhage (IVH) as well as combinations of those.

In all patients, an initial CT scan was performed as well as a follow-up CT within $24 \mathrm{~h}$. In the stroke patients, the average National Institute of Health Stroke Scale (NIHSS) was $14 \pm 7$. Three patients were intubated prior to their arrival at the emergency unit; therefore, NIHSS assessment was not possible. Patients suffered from vascular occlusion of the MCA (M1 segment $40 \%$, M2 segment $4 \%$ ), the basilar artery (22\%), the ICA bifurcation $(6 \%)$, the vertebral artery (14\%) and the ICA (14\%). FD-CT was performed directly before starting EMT and after IV rt-PA therapy had been initiated immediately after CT to exclude a new secondary intracranial bleeding. In the patients with ICH, FD-CT was performed on neurosurgical standby because the patients presented clinical deterioration indicative for post-hemorrhagic hydrocephalus, and using FD-CT offers the option to implant an external ventricular drainage directly within the Angio Suite without transferring the patient to the operating room. FD-CT was performed in the TIA patients directly before stenting of a symptomatic vessel stenosis to exclude any hemorrhagic alteration after fast initiation of dual anti-aggregation therapy. The average time interval between FD-CT and regular CT was $1.57 \mathrm{~h}$ in the stroke patients, $12.62 \mathrm{~h}$ in the patients with ICH and $31.89 \mathrm{~h}$ in the TIA patients.

SAHs were assessed according to the Fisher grading scale from $1^{\circ}$ to $5^{\circ}[16]$, and the patients' clinical status according to the Hunt and Hess scale. Perimesencephalic SAH is characterized by hemorrhage restricted to the perimesencephalic or prepontine cisterns with minimal extension into the ambient and quadrigeminal cisterns and to the medial part of the Sylvian fissures and none to the intraventricular space.

\section{Flat-Detector CT}

Image acquisition was performed on a biplane FD angiographic system (Axiom Artis dBA, Siemens Healthcare GmbH, Forchheim, Germany) using a standard program as provided by the manufacturer (20sDR-H program 'DynaCT': 20-second rotation, projection on $30 \times 40 \mathrm{~cm}$ detector size, $217^{\circ}$ angular range, increment $0.4^{\circ}$ /image). Technical details of the program have been published and described elsewhere [13-15]. The average acquisition time including image reconstruction is about $1 \mathrm{~min}$.

\section{Post-Processing}

All data sets were reconstructed post hoc using the following parameters: kernel type 'HU', image characteristics 'smooth' and reconstruction mode 'native fill'. In addition, the newly implemented half-scan cone-beam algorithm 'DynaCT Head clear' (Siemens Healthcare GmbH, Forchheim, Germany) was applied in all cases. This algorithm uses the method of Grangeat [17] and a linear interpolation technique [18] in addition to adjustment and correction of ring artifacts, beam hardening, scattered radiation 
and truncated projections. This technique improves the image quality in peripheral slices of the data volume, for example, in the skull base, compared with the previous 'Feldkamp-Davis-Kress (FDK) algorithm' (DynaCT Head, Siemens Healthcare GmbH, Forchheim, Germany). While by the 'FDK algorithm', only approximations for the peripheral slices are generated, exact computations of these slices are provided by the Gangreat-based 'DynaCT Head clear' algorithm.

After image reconstruction, a volume data set with about 400 slices in a $512 \times 512$ matrix is available. Axial MPRs with $5 \mathrm{~mm}$ slice thickness and spacing were reconstructed, covering the entire brain. After anonymization, the MPRs were stored in random order for evaluation.

\section{Data Analysis}

Two experienced neuroradiologists (RAD1/RAD2), unaware of clinical findings, evaluated independently the FD-CTs. During analysis, the reader was allowed to adapt image windowing, since there is no standardization. The windowing values of FD-CT vary between 150-250 HU in width and 50-100 HU in center. First, both raters had to evaluate the quality of the data (good, slightly compromised or strongly compromised), the presence of artifacts (i.e., ring, motion, beam hardening artifacts) and had to assess whether the image quality is sufficient for evaluation. Next, they had to screen the images for hemorrhage or ischemic signs. In case of a hemorrhage, the raters had to differentiate between ICH, IVH, $\mathrm{SAH}$ or a combination of these. Additionally, the location of the hemorrhage had to be described.

The findings of the raters were correlated with CT which served as reference standard, evaluated by a third, experienced neuroradiologist, not involved in FD-CT evaluation. All CT examinations were performed on a 128-section CT scanner (Somatom Definition AS+, Siemens Healthcare GmbH, Forchheim, Germany). The acquisition was performed using the following parameters: collimation $0.6 \mathrm{~mm}$, increment $0.4 \mathrm{~mm}$ and reconstruction kernel H31s. From that data, axial image reconstructions with a slice thickness of $4.8 \mathrm{~mm}$ were generated.

\section{Statistics}

Statistical analysis was performed using SPSS 20.0. Rater agreement to CT, regarding hemorrhage or infarction detection, was assessed by calculating the Pearson's correlation coefficient ( $r$ ). The significance level $\mathrm{p}$ was set at $\mathrm{p} \leq 0.05$. Inter-rater agreement was calculated using the Cohen's kappa coefficient $(\kappa)$. A value of 0.41-0.60 for the $\kappa$-coefficient was interpreted as moderate agreement, a value of $0.61-0.80$ as substantial agreement and a value of $0.81-1.00$ as almost perfect agreement.

\section{Results}

\section{Image Quality}

Out of the 102 patients, RAD1 excluded no and RAD2 one data set, because of an insufficient image quality, resulting from motion artifacts. RAD1 assessed 9\% $(n=9)$ and RAD2 $18 \%(\mathrm{n}=18)$ of the FD-CTs as slightly compromised in image quality (by motion-, ring- or beamhardening artifacts). Three percent $(n=3$ or $n=4$, respec-
Table 1. Distribution of different types of hemorrhage within study population

\begin{tabular}{lr}
\hline Hemorrhage & $\mathrm{n}$ \\
\hline Patients with hemorrhage & 45 \\
ICH & 21 \\
With additional IVH & 1 \\
SAH & 6 \\
With additional IVH & 2 \\
With additional ICH & 3 \\
With additional ICH and IVH & 1 \\
Perimesencephalic & 10 \\
IVH & 1 \\
Total number of hemorrhages & 55 \\
ICH & 28 \\
SAH & 22 \\
IVH & 5 \\
\hline
\end{tabular}

tively) of the scans were assessed as strongly compromised but still diagnostic in image quality by both raters resulting from motion artifacts.

\section{Hemorrhage Detection}

Eight patients showed more than one location or type of hemorrhage, so the total number of hemorrhages was 55 (table 1).

\section{Primary Intracerebral Hemorrhage}

Both raters detected all 22 primary ICH correctly (compare fig. 1), except one infratentorial cerebellar ICH, which was missed by RAD1 (compare fig. 2c, f), meaning a sensitivity of $95 \%$ for RAD1 and of $100 \%$ for RAD2. No ICH was diagnosed 'false-positive', meaning a specificity of $100 \%$ for both raters. In 1 case, ICH had additional intraventricular extension, and the associated IVB was detected correctly by both raters. If only supratentorial ICH are considered, the sensitivity and specificity of ICH detection can be assessed as $100 \%$ each for both raters.

\section{Primary IVH}

The one primary IVH was detected correctly by both raters, meaning a sensitivity and specificity of $100 \%$ each (compare fig. 2a, d). No 'false-positive' IVH was reported.

\section{Subarachnoid, Non-Perimesencephalic Hemorrhage}

RAD1 detected 9, RAD2 8 of the 12 non-perimesencephalic SAH correctly (compare fig. $2 \mathrm{~b}$, e). Both rates missed the identical 3 supratentorial SAH. All these SAH were classified as Fisher $2^{\circ}$, and all these patients presented clinically as Hunt and Hess $1^{\circ}$. The 1 supratentorial SAH, 


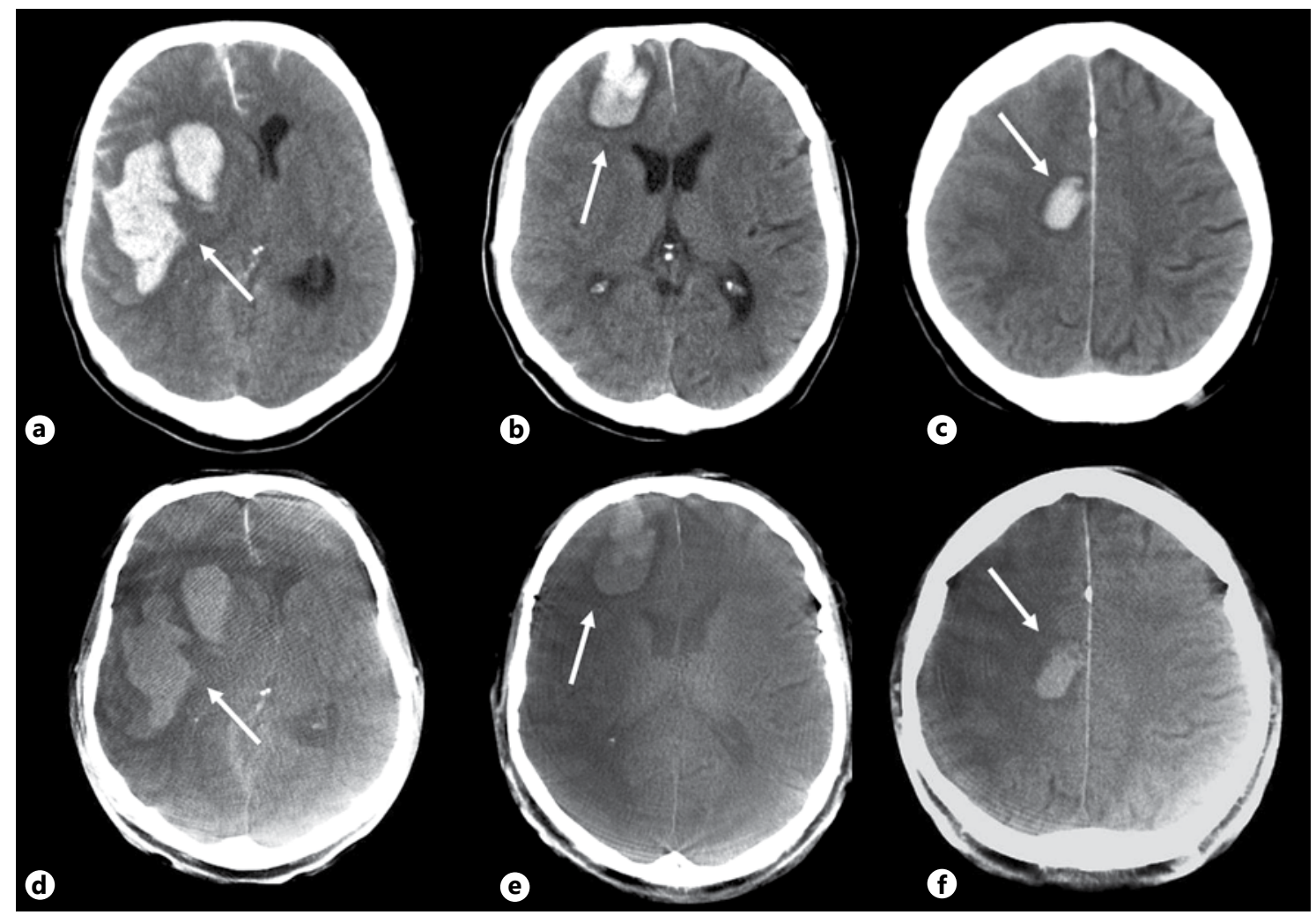

Fig. 1. a-c Show cranial CT of 3 patients with supratentorial ICH (marked by white arrow, respectively) located in the right basal ganglia/temporal lobe (a), and the right frontal lobe (b and $\mathbf{c}$ ). d-f Present the corresponding FD-CTs allowing hemorrhage delineation in a comparable way.

which was additionally missed by RAD2, was classified as Fisher $3^{\circ}$ and was located in the central sulcus, presenting clinically as Hunt and Hess $1^{\circ}$. Associated ICH and IVH were detected correctly in all 6 cases by both raters. The sensitivity of detecting non-perimesencephalic SAH was $75 \%$ for RAD 1 and $67 \%$ for RAD2. No 'false-positive' SAH was reported, meaning a specificity of $100 \%$ for both raters.

Perimesencephalic Hemorrhage

Both raters missed the identical 7 of the 10 non-aneurysmal perimesencephalic SAH. All perimesencephalic SAH were classified as Fisher $2^{\circ}$, and the patient presented clinically as Hunt and Hess $1^{\circ}$. Concerning perimesencephalic SAH detection, the sensitivity was $30 \%$ and the specificity $100 \%$ for both raters.

Overall, 44 of the $55 \mathrm{ICHs}$ were correctly detected by both raters, meaning a sensitivity of $80 \%$ and a specificity of $100 \%$ for the detection of any ICH. Inter-rater agreement $(\kappa=0.92,95 \%$ CI $0.837-0.997, \mathrm{p} \leq 0.001)$ turned out as almost perfect. Rater agreement to CT (RAD1: $r=0.81$, RAD2: $r=0.81, p \leq 0.001$ ) was statistically significant.
If SAH are excluded from statistical analysis, since they can be considered a separate clinical entity presenting with clinical symptoms differing from ischemic stroke or $\mathrm{ICH}$, then the sensitivity of hemorrhage detection increases to $97-100 \%$ with a specificity of $100 \%$.

\section{Ischemic Signs}

Acute Infarct Demarcation

Four of the 32 acute, ischemic stroke patients showed early signs of stroke in the initial CT scan in sense of an obscuration of the grey-white matter junction, but they were selected for EMT based on a mismatch concept regarding perfusion information. In 1 case, these early signs of stroke were detected correctly in FD-CT by only one rater, but in the other cases, these signs were missed by both raters. In 2 cases, an acute infarction was supposed by both readers false-positively, meaning a sensitivity of 0 or $25 \%$ respectively, and a specificity of $98 \%$. In one of these 2 cases, RAD1 assessed image quality as strongly compromised due to motion artifacts. In 7 cases, a 'hyperdense media sign' was delineated in the CT scan, but not detected in the FD-CT.
Eckert/Gölitz/Lücking/Struffert/ Knossalla/Doerfler 


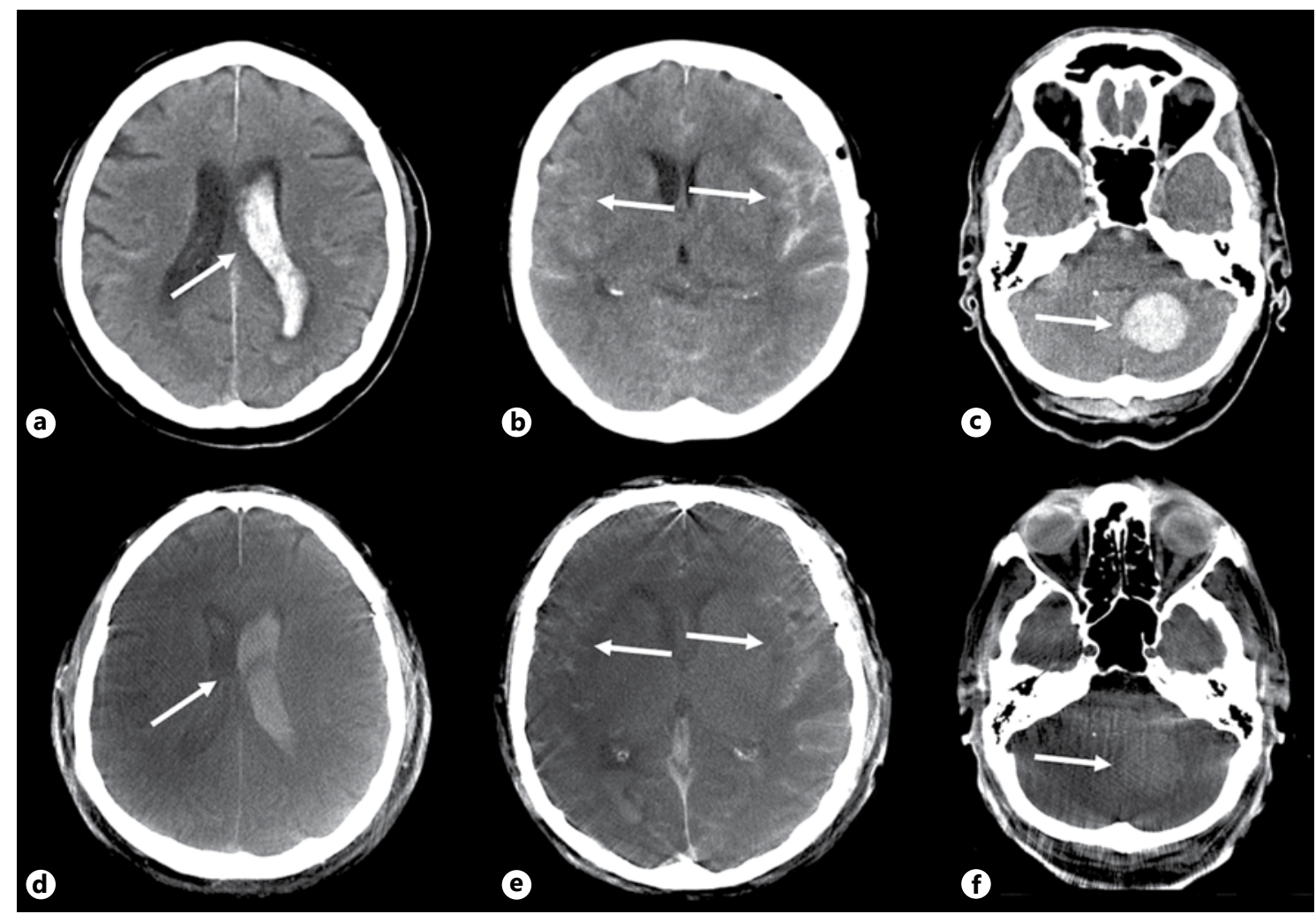

Fig. 2. a-c Demonstrate cranial CT of 3 patients with IVH in the left, lateral ventricle (a), with bihemispheric SAH (b), and with infratentorial ICH in the left cerebellum (c) (marked by white arrow, respectively). In the lower row, the corresponding FD-CTs are shown allowing IVH and SAH assessment equally to conventional CT ( $\mathbf{d}$ and $\mathbf{e}$ ). The cerebellar ICH is only faintly delineable in the FD-CT and was missed therefore by RAD1 (f).

Suite [3]. If FD-CT could replace conventional multislice $\mathrm{CT}$, acute stroke patients may be referred directly to the Angio Suite for diagnostic imaging and endovascular treatment. This would avoid patient transport from one modality to the other, and would thereby save time, increase patient safety and improve patient outcome. A requirement to implement multimodal FD-CT as an initial stroke imaging tool is the reliable hemorrhage exclusion $[12,19]$.

Our retrospective study in 102 patients aimed to verify that FD-CT can exclude cerebral hemorrhage reliably in stroke patients. Moreover, the potential of FD-CT in detecting signs of infarction in acute, stroke patients was evaluated.

Previous studies [13-15], investigating hemorrhage visualization in FD-CT using the FDK algorithm, found limitations in hemorrhage detection near skull base because of motion- and beam-hardening artifacts, as well as an inferiority to conventional CT concerning SAH delineation. However, all these studies are limited by their 


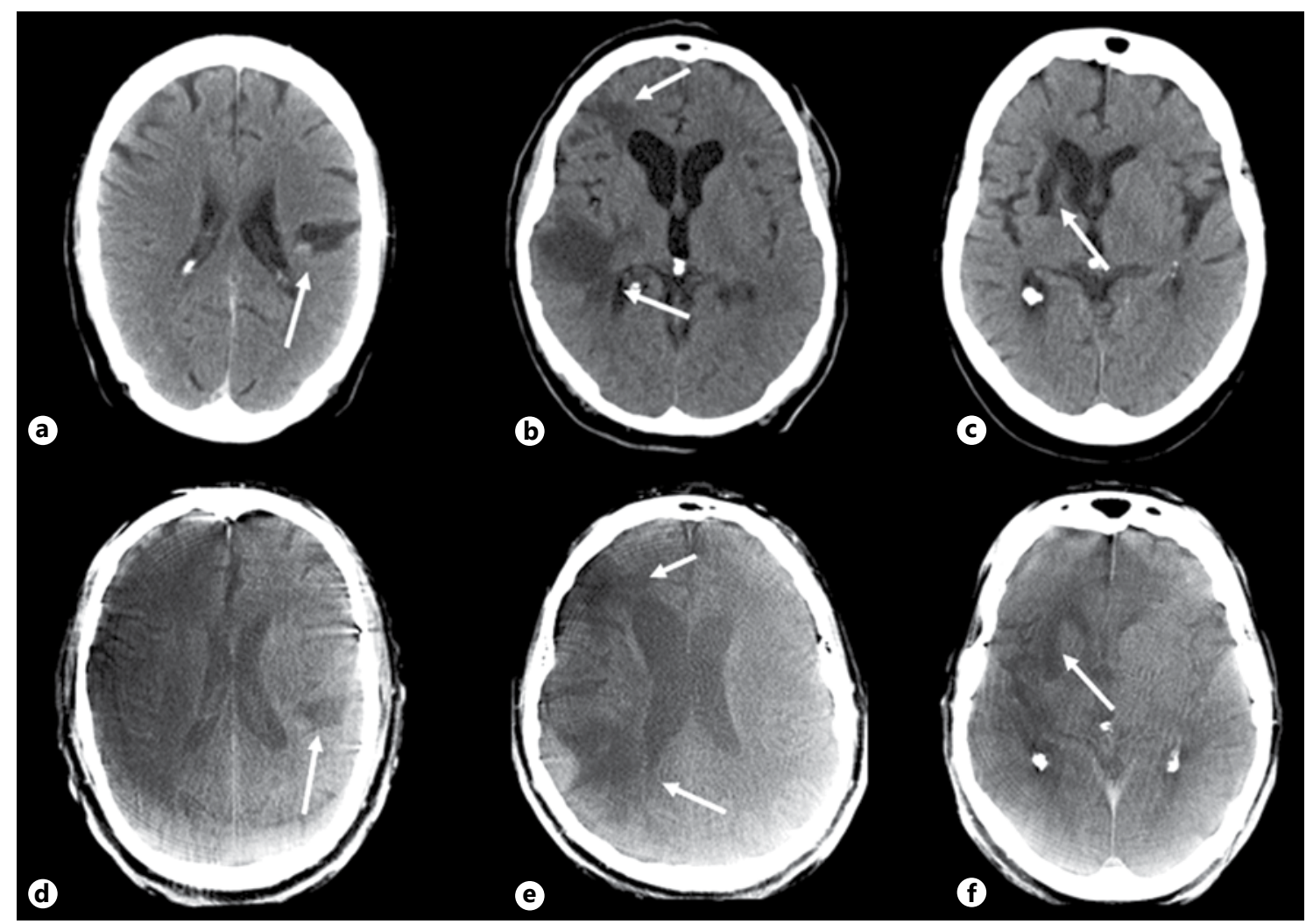

Fig. 3. a-c Illustrate chronic post-ischemic parenchymal defects in cranial CT, located left frontal (a), right temporal and right frontal (b) and in the right nucleus lentiformis (c; marked by white arrow, respectively). In the corresponding FD-CTs (d-f), the post-ischemic defects can be revealed in a comparable way.

small sample size. Additionally, up to now, there is no available data using the newly implemented half-scan cone-beam algorithm regarding hemorrhage detection. Moreover, the potential to detect infarction signs in native FD-CT has not yet been evaluated.

Based on our results, FD-CT can be considered a reliable tool to exclude supratentorial ICH and IVH demonstrating a sensitivity and specificity of $100 \%$ each. On the other hand, FD-CT has limitations in the detection of infratentorial ICH probably due to its low soft tissue resolution. In consequence, in our study, one in the posterior fossa located ICH was missed by one rater. Moreover, FD-CT demonstrated limitations in the detection of SAH (sensitivity 67-75\%), especially an SAH with less than $1 \mathrm{~mm}$ thickness and perimesencephalic SAH (sensitivity $30 \%$ ). Thus, both raters missed 10 Fisher $2^{\circ} \mathrm{SAH}$, which presented clinically as a Hunt and Hess $1^{\circ}$ and of whom 7 were perimesencephalic. Besides the lower contrast resolution, the presence of beam hardening artifacts in the brain stem region may explain these FD-CT limitations [20, 21]. Even the use of the newly imple- mented post-processing algorithm does not seem to solve these FD-CT restrictions. Especially, compromising motion artifacts, which were the most observed artifacts in our study and affected the entire data set and not just single slices, will remain a known problem in FD-CT imaging [14] despite the 'DynaCT Head clear' algorithm.

In our opinion, the low sensitivity of SAH detection by FD-CT should not impede the use of multimodal FD-CT in general. The clinical presentation of SAH patients, especially of those with perimesencephalic SAH, is different to those suffering from ischemic stroke with large vessel occlusion. On the other side, in patients with typical symptoms of acute ischemic stroke, ICH is the main diagnosis to be excluded. All cases without hemorrhage were assessed correctly by the raters. The high inter-rater agreement $(\kappa=0.92)$ regarding ICH detection indicates that FD-CT evaluation is not rater-dependent. Also, the rater agreement to $\mathrm{CT}$ regarding $\mathrm{ICH}$ detection turned out as highly significant. Therefore, in our experience, especially ICH can be detected reliably. Neverthe- 
less, since missing small or infratentorial hemorrhage as well as SAH without parenchymal clot could have disastrous sequelae, FD-CT imaging does not seem ready for wide adoption yet or for replacing regular CT, and should be reserved for selected patients, taking into account not only its limitations but also its potential in speeding up the workflow that patients can benefit from. To compensate this drawback, in emergency cases, a multimodal approach including FD-CT angiography and FD-CT perfusion seems mandatory to confirm large vessel occlusion or perfusion deficit before therapy initiation.

In detecting signs of infarction, FD-CT is known to be limited due to its lower contrast resolution of approximately 10 Hounsfield units as compared to conventional CT [22]. Correspondingly, in our study, the sensitivity of detecting early signs of stroke turned out as poor (0-25\%). In accordance, defining on FD-CT, the Alberta Stroke Program Early CT score (ASPECTS) as an important prognostic parameter before EMT is limited too. Thus, plain FD-CT seems to be insufficient as standalone modality in acute stroke needing adjunct imaging. Because of the small size of early signs of stroke in our study population, these results can be considered only preliminary. A step to improve the detection of ischemic stroke could be the use of multimodal functional FD-CT imaging $[23,24]$. We assume that by additive FD-CT angiography and FD-CT perfusion imaging, the sensitivity and specificity in the absence of early ischemic changes may be dramatically improved, comparably as described for conventional CT in the detection of acute ischemic stroke [4]. Especially, the implementation of new post-processing algorithms seems to improve functional FD-CT imaging further, allowing the generation of angiography and perfusion images with comparable quality to CT angiography and CT perfusion $[25,26]$. In accordance to other authors $[27,28]$, attaching value to multimodal imaging of the ischemic penumbra, we believe that this functional imaging is the key to recognize (large vessel occlusion, perfusion deficit) and initiate ischemic stroke therapy, and not the recognition of early ischemic signs difficultly to delineate. In our opinion, this is supported by current stroke studies that turned out positively on the basis of recognition of large vessel occlusion and, in some, on mismatch evaluation. To select patients for EMT based on perfusion imaging offers the option of a more individualized approach than using only generalized time criteria, especially since the timeline for conversion of ischemic penumbra into infarct tissue may be individually different. Accordingly, in the
EXTEND-IA trial, CT perfusion was used to select patients for EMT, achieving an impressive rate of good clinical outcome at $71 \%$ by this approach [4]. Moreover, the assessment of the leptomeningeal collateral status seems to be an important parameter in acute ischemic stroke, since good pre-treatment collaterals may enhance the rates of successful reperfusion and recanalization in endovascular treatment [29]. On the other hand, using plain head CT and the ASPECTS score have been proven to work well, and it is faster than a multimodal approach. Additionally, it needs less radiation dose and no contrast medium exposure to the patient. Maybe, the sensitivity of detecting early stroke signs on FD-CT might increase if instead of blinded readings the raters would be informed about clinical symptoms what is crucial to be known for image evaluation in stroke patients. Whether the sensitivity will indeed increase by knowing the clinical background could be an objective for further investigations.

Certainly, our study has some limitations. First, the study has low sample size of infratentorial ICH and early stroke signs. In addition, there was no $\mathrm{PH}$ or IVH smaller than $5 \times 10 \mathrm{~mm}$ included in our study. Furthermore, prospective investigations with an increased sample size are desirable to elucidate the reliability of FD-CT imaging in the detection of infratentorial ICH and acute infarction signs. The use of special head-fixing cups, which prevent head movement and therefore motion artifacts, could probably contribute to image improvement. Moreover, detector optimization and software innovations concerning artifact filtering and improvement of the low contrast resolution will be beneficial to overcome the limitations of FD-CT imaging.

\section{Conclusions}

Based on our results, FD-CT using a new post-processing technique seems to be a reliable tool for the detection of supratentorial ICH and IVH. On the other hand, the reliability concerning the detection of perimesencephalic SAH as well as infratentorial ICH turned out as moderate. The potential of FD-CT in detecting ischemic changes seems limited, especially if evaluated unaware of clinical findings. Thus, plain FD-CT seems insufficient as a standalone modality in acute stroke, but when embedded within a multimodal functional approach, FD-CT imaging may be capable of reliably detecting ischemic stroke patients and exclude ICH in selected cases taking clinical information into account. For further evaluations, a com- 
parative study between FD-CT and regular CT should be performed, investigating prospectively the reliability of FD-CT in stroke imaging.

\section{Acknowledgments}

The present work was performed in fulfillment of the requirements for obtaining the degree Doctor od Medicine at the Friedrich-Alexander-University Erlangen-Nuremberg.

\section{Funding}

The authors gratefully acknowledge funding of the Medical Valley national leading edge cluster, Erlangen, Germany, diagnostic imaging network, sub-project $\mathrm{BD} 16$, research grant No. 13EX1212G and sub-project BD 20, research grant No 13G 0031A.

\section{Disclosure Statement}

The authors declare no conflict of interest.

\section{References}

1 Berkhemer OA, Fransen PS, Beumer D, et al: A randomized trial of intraarterial treatment for acute ischemic stroke. N Engl J Med 2015; 372:11-20.

2 Goyal M, Demchuk AM, Menon BK, et al: Randomized assessment of rapid endovascular treatment of ischemic stroke. N Engl J Med 2015;372:1019-1030

3 Campbell BC, Mitchell PJ, Kleinig TJ, et al: Endovascular therapy for ischemic stroke with perfusion-imaging selection. $\mathrm{N}$ Engl $\mathrm{J}$ Med 2015;372:1009-1018.

4 Kloska SP, Nabavi DG, Gaus C, et al: Acute stroke assessment with CT: do we need multimodal evaluation? Radiology 2004;233:7986.

5 Scharf J, Brockmann MA, Daffertshofer M, et al: Improvement of sensitivity and interrater reliability to detect acute stroke by dynamic perfusion computed tomography and computed tomography angiography. J Comput Assist Tomogr 2006;30:105-110.

6 Broderick JP, Palesch YY, Demchuk AM, et al: Endovascular therapy after intravenous t-PA versus t-PA alone for stroke. $\mathrm{N}$ Engl J Med 2013;368:893-903.

7 Khatri P, Abruzzo T, Yeatts SD, et al: Good clinical outcome after ischemic stroke with successful revascularization is time-dependent. Neurology 2009;73:1066-1072.

8 Jovin TG, Chamorro A, Cobo E, et al: Thrombectomy within 8 hours after symptom onset in ischemic stroke. N Engl J Med 2015;372: 2296-2306.

9 Gupta R, Cheung AC, Bartling SH, et al: Flatpanel volume CT: fundamental principles, technology, and applications. Radiographics 2008;28:2009-2022.

10 Kau T, Hauser M, Obmann SM, et al: Flat detector angio-CT following intra-arterial therapy of acute ischemic stroke: identification of hemorrhage and distinction from contrast accumulation due to blood-brain barrier disruption. AJNR Am J Neuroradiol 2014;35: 1759-1764.
11 Struffert T, Deuerling-Zheng Y, Kloska S, et al: Flat detector CT in the evaluation of brain parenchyma, intracranial vasculature, and cerebral blood volume: a pilot study in patients with acute symptoms of cerebral ischemia. AJNR Am J Neuroradiol 2010;31:1462-1469.

12 Jauch EC, Saver JL, Adams HP Jr, et al: Guidelines for the early management of patients with acute ischemic stroke: a guideline for healthcare professionals from the American Heart Association/American Stroke Association. Stroke 2013;44:870-947.

13 Doelken M, Struffert T, Richter G, et al: Flatpanel detector volumetric CT for visualization of subarachnoid hemorrhage and ventricles: preliminary results compared to conventional CT. Neuroradiology 2008;50:517-523.

14 Struffert T, Eyupoglu IY, Huttner HB, et al: Clinical evaluation of flat-panel detector compared with multislice computed tomography in 65 patients with acute intracranial hemorrhage: initial results. Clinical article. J Neurosurg 2010;113:901-907.

15 Struffert T, Richter G, Engelhorn T, et al: Visualisation of intracerebral haemorrhage with flat-detector CT compared to multislice CT: results in 44 cases. Eur Radiol 2009;19:619_ 625.

16 Hunt WE, Hess RM: Surgical risk as related to time of intervention in the repair of intracranial aneurysms. J Neurosurg 1968;28:14-20.

17 Grangeat P: Mathematical framework of cone beam $3 \mathrm{D}$ reconstruction via the first derivative of the radon transform; in Herman G, Louis A, Natterer F (eds): Mathematical Methods in Tomography. Berlin Heidelberg, Springer, 1991, vol 1497, pp 66-97.

18 Lee SW, Wang G: Grangeat-type helical halfscan computerized tomography algorithm for reconstruction of a short object. Med Phys 2004;31:4-16.

19 Norris JW, Buchan A, Cote R, et al: Canadian guidelines for intravenous thrombolytic treatment in acute stroke. A consensus statement of the Canadian stroke consortium. Can J Neurol Sci 1998;25:257-259.

20 Kalender WA: Der Einsatz von Flachbilddetektoren für die CT-Bildgebung. Radiologe 2003;43:379-387.
21 Gupta R, Cheung AC, Bartling SH, et al: Flatpanel volume CT: fundamental principles, technology, and applications. Radiographics 2008;28:2009-2022.

22 Loose R, Wucherer M, Brunner T: Visualization of 3D low contrast objects by CT conebeam reconstruction of a rotational angiography with a dynamic solid body detector. RoFo 2005;S1:PO160.

23 Struffert T, Deuerling-Zheng Y, Engelhorn T, et al: Feasibility of cerebral blood volume mapping by flat panel detector CT in the angiography suite: first experience in patients with acute middle cerebral artery occlusions. AJNR Am J Neuroradiol 2012;33:618-625.

24 Struffert T, Deuerling-Zheng Y, Kloska S, et al: Dynamic angiography and perfusion imaging using flat detector $\mathrm{CT}$ in the angiography suite: a pilot study in patients with acute middle cerebral artery occlusions. AJNR Am J Neuroradiol 2015;36:1964-1970.

25 Yang P, Niu K, Wu Y, et al: Time-resolved Carm computed tomographic angiography derived from computed tomographic perfusion acquisition: new capability for one-stop-shop acute ischemic stroke treatment in the angiosuite. Stroke 2015;46:3383-3389.

26 Niu K, Yang P, Wu Y, et al: C-arm conebeam $\mathrm{CT}$ perfusion imaging in the angiographic suite: a comparison with multidetector CT perfusion imaging. AJNR Am J Neuroradiol 2016;37:1303-1309.

27 Davis S, Donnan GA: Time is Penumbra: imaging, selection and outcome. The Johann jacob wepfer award 2014. Cerebrovasc Dis 2014;38:59-72.

28 Gory B, Riva R, Derex L, Nighoghossian N, Turjman F: Value of perfusion CT-guided recanalization therapy in acute ischemic stroke patients. Cerebrovasc Dis 2014;37:389-390.

29 Leng X, Fang H, Leung TW, et al: Impact of collateral status on successful revascularization in endovascular treatment: a systematic review and meta-analysis. Cerebrovasc Dis 2016;41:27-34 